\section{Scoring insertion-deletion polymorphisms by dynamic allele-specific hybridization}

\author{
Sarah L. Sawyer, W. Mathias Howell, and Anthony J. Brookes \\ Karolinska Institute, Stockholm, Sweden
}

BioTechniques 35:292-298 (August 2003)

Genome variation provides researchers with thousands of markers with which to study human demographic history and phenotypes. Insertion-deletion (indel) polymorphism is an important and abundant form of human genome variation, and convenient methods for genotyping indels are therefore needed. Here we evaluate dynamic allele-specific hybridization (DASH) for its ability to score indels. Evaluation of six model indel DASH assays based on synthetic oligonucleotides showed that length differences of 1-5 bp were accurately scored. Only single probes were required to assay indels of 3-4 bp or less, while longer indels tended to require the use of both allele probes serially. The best results were obtained by central placing of the probe over the indel. Model study findings were confirmed by running indel DASH assays upon PCR-amplified targets representing four polymorphisms from Alzheimer's disease candidate genes APBB1 and LRP1. These indels were genotyped in a set of 121 patients and 156 controls. While no disease association was found, the data quality confirmed that DASH is a robust and useful procedure for genotyping indels of the size range typically found in the human genome.

\section{INTRODUCTION}

Across academic and commercial sectors, a massive research undertaking is under way to address the identity, technologies, and utility of human genome variation. Single nucleotide polymorphisms (SNPs) are particularly emphasized because of their abundance and potential ease of genotyping. However, a second valuable form of polymorphism is the insertion-deletion (indel) polymorphism. Like SNPs, indels may be used as markers in the search for genetic risk factors that influence disease and also as resources for population genetics and evolutionary studies. Diallelic indels are abundant, accounting for approximately $8 \%$ of all human DNA sequence variants (1). Analysis of human chromosome 22 showed that $18 \%$ of that chromosome's sequence variation is indel polymorphism, with more that half of this involving simple 1-bp variants (2). Likewise, in the Human Genomic Variation Database (HGVbase; http://hgvbase. cgb.ki.se) (3), there are 36,959 indels, $87 \%$ of which are between 1 and $5 \mathrm{bp}$ in length, and $76 \%$ are from 1 to $3 \mathrm{bp}$. Therefore, a system that could robustly genotype $1-5$ bp indels would repre- sent a very useful technology for human genome analysis.

Here we have used a range of synthetic oligonucleotide targets and targets derived by PCR to evaluate the use of an established SNP scoring system called dynamic allele-specific hybridization (DASH) (4) for genotyping 1 - to 5-bp indels. Our findings demonstrated robust assay functionality, which we have exploited to Alzheimer's disease association analysis upon four different candidate gene indels. No disease associations were revealed, but the uniformity and quality of the accrued data endorse DASH as a highly effective method for indel genotyping.

\section{MATERIALS AND METHODS}

\section{Oligonucleotides}

All oligonucleotides were synthesized and high-performance liquid chromatography (HPLC)-purified by Thermo Hybaid (Ulm, Germany).

\section{Clinical Materials}

Patient DNA was obtained from 121 nonfamilial early-onset Alzheimer's disease cases listed in the Lothian Psychiatric Case Register, from the Scottish population, as previously described (5). Control samples were from church congregation volunteers in the Lothian Region. All controls met the MiniMental State Examination exclusion criteria for dementia (6).

\section{PCR}

For experiments involving PCR-amplified products, primers were designed using Oligo 6.0 software (Molecular Biology Insights, Cascade, CO, USA) to amplify a 50- to 70-bp fragment spanning the indel of interest. One primer for each assay design was synthesized to carry a 5'-biotin label. Each 25- $\mu$ L PCR mixture contained 5-10 ng genomic DNA, 3 pmol biotinylated primer, 15 pmol nonbiotinylated primer, $0.75 \mathrm{U}$ AmpliTaq Gold ${ }^{\circledR}$ (Applied Biosystems, Foster City, CA, USA), $10 \%$ dimethyl sulfoxide (DMSO), $1 \times$ AmpliTaq Gold Buffer including 1.5 $\mathrm{mM} \mathrm{MgCl} 2$ (Applied Biosystems), and $0.2 \mathrm{mM}$ each dNTP. Thermal cycling conditions were as follows: $10 \mathrm{~min}$ at $94^{\circ} \mathrm{C}$, followed by 40 cycles of $15 \mathrm{~s}$ at $94^{\circ} \mathrm{C}$ and $30 \mathrm{~s}$ at $51^{\circ} \mathrm{C}, 53^{\circ} \mathrm{C}$, or $55^{\circ} \mathrm{C}$. PCR thermal cycling was done in 96well microplates on a Touchdown ${ }^{\mathrm{TM}}$ temperature cycling apparatus (Thermo Hybaid, Ashford, UK). To verify successful amplification, $3 \mu \mathrm{L}$ of four randomly chosen samples were examined on a $3 \%$ low-melt agarose gel.

\section{DASH}

DASH was performed as previously described (7). In brief, biotinylated targets (either synthesized or PCR-amplified) were immobilized in wells of a streptavidin-coated microplate. For synthetic targets, this involved mixing 5 pmol oligonucleotide target with $25 \mu \mathrm{L}$ HEN buffer (0.1 M HEPES, pH 8.0, 10 $\mathrm{mM}$ EDTA, $50 \mathrm{mM} \mathrm{NaCl}$ ) and transferring to the well. For PCR-amplified targets, the PCR product was diluted to 50 $\mu \mathrm{L}$ with HEN followed by transfer of $25 \mu \mathrm{L}$ solution to a binding plate. After a minimum of $1 \mathrm{~h}$ of binding time at room temperature, the transferred solution was removed, and the wells were rinsed with $50 \mu \mathrm{L} 0.1 \mathrm{M} \mathrm{NaOH}$ to denature the PCR products. Then, $50 \mu \mathrm{L}$ 
HEN buffer containing 30 pmol of the appropriate probe was added to each well. Duplex denaturation was determined by monitoring fluorescence generated either through the simple use of SYBR ${ }^{\circledR}$ Green I fluorescent dye (Molecular Probes, Leiden, The Netherlands) or through induced fluorescence resonance energy transfer (iFRET) involving 3'-ROX-labeled probes for centrally positioned probes; all other probes were unlabeled. The samples were heated to $80^{\circ} \mathrm{C}$ for $1 \mathrm{~min}$ and allowed to cool to room temperature to take probe hybridization to completion. Finally, the hybridization solution was discarded and replaced with $50 \mu \mathrm{L}$ HEN buffer containing $0.01 \%$ SYBR Green I fluorescent dye. Subsequently, the assay

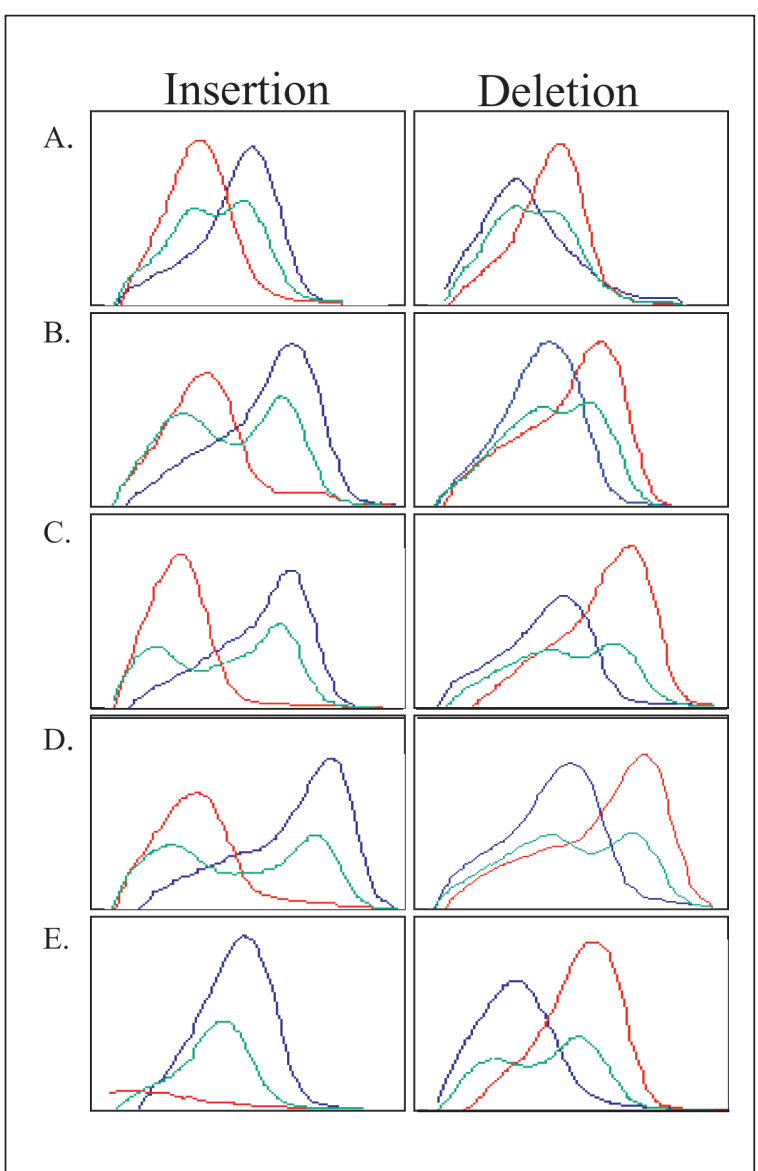

Figure 1. DASH results for the synthetic indels. Results for IND000000167 are not shown but are essentially equivalent to IND000003300. The genotyping results are as follows: red, homozygous insertion allele; blue, homozygous deletion allele; and green, heterozygous mixture of alleles. The $\mathrm{x}$-axis is temperature, $35^{\circ}-80^{\circ} \mathrm{C}$. The $y$-axis is $-\mathrm{dFluorescence} / \mathrm{dTemperature}$. The following are indel IDs followed by size: (A) IND000003300, 1 bp; (B) IND000003650, 2 bp; (C) IND000005021, 3 bp; (D) IND000007079, 4 bp; and (E) IND000002709, 5 bp. plates were examined on a melting curve analysis machine (Thermo Hybaid). The software was programmed to heat the samples from $35^{\circ} \mathrm{C}$ to $80^{\circ} \mathrm{C}$ at a rate of $0.3^{\circ} \mathrm{C} / \mathrm{s}$ while continually measuring fluorescence. Fluorescence changes (DNA melting profiles) were compared by plotting the negative derivative of fluorescence against temperdenaturation rate [target-probe melting temperatures $\left.\left(\mathrm{T}_{\mathrm{m}} \mathrm{s}\right)\right]$.

\section{RESULTS AND DISCUSSION}

In brief, DASH genotyping entails (i) creating short single-stranded representations of a sample DNA (typically via PCR) spanning the sequence to be genotyped; (ii) immobilization of these target molecules to a surface; (iii) annealing a known probe sequence that is perfectly complementary to one of the possible alleles (achieving saturation hybridization, regardless of whether the probe stably matches or somewhat less stably mismatches the target); (iv) steadily heating the probe-target duplex while following the denaturation process in real time; and $(v)$ comparing the temperatures at which denaturation rates for different samples are maximal $\left(\mathrm{T}_{\mathrm{m}} \mathrm{s}\right)$, to indicate their relative stabilities and thereby reveal which allele(s) were present in the tested DNAs.

\section{Model Experiments- Basic Functionality}

To assess whether DASH could discriminate short insertion and deletion alleles, we first set up a series of control experiments involving model assays based on synthetic oligonucleotide targets. Indels of 1, 2, 3, 4, and 5 bp in size were chosen for study, representing real polymorphisms listed in HGVbase. These polymorphisms, along with the synthesized targets and probes, are detailed in Table 1. Two probes and two targets were created for each modeled polymorphism, representing the insertion allele and the deletion allele versions of each reactant. For these initial studies, the probes were labeled with a 3 -ROX moiety and designed with the indel sequence located centrally along the length of the probe.

The target molecules carried 5'-biotin moieties for physical immobilization of the different allele copies, representing the homozygous genotypes, of the single-stranded targets in different wells of a streptavidin-coated microplate. Additionally, a 1:1 mixture of insertion and deletion allele targets was bound to microplate wells to mimic heterozygous DNA samples. The different target wells were all prepared as replicates of at least four. Half of these were dynamically probed by DASH with the insertion allele probe, and the other half with the deletion allele probe.

The DASH results for these experiments showed that for 1-, 2-, 3-, and 4 bp indels, a single denaturation $\left(\mathrm{T}_{\mathrm{m}}\right)$ peak was created when the insertion probe or the deletion probe was used to interrogate either the insertion target or the deletion target (Figure 1). For each probe, the observed $\mathrm{T}_{\mathrm{m}} \mathrm{s}$ were highly reproducible $\left(<1{ }^{\circ} \mathrm{C}\right.$ variance; Figure 2$)$, and the $\mathrm{T}_{\mathrm{m}}$ of the matched target-probe duplex was between $6^{\circ}$ and $16^{\circ} \mathrm{C}$ higher than the $\mathrm{T}_{\mathrm{m}}$ of the mismatched targetprobe duplex (larger difference for bigger deletions). For the artificial heterozygote targets, double $T_{m}$ peaks were observed made up of individual $\mathrm{T}_{\mathrm{m}} \mathrm{s}$ that reflected those seen for the component "homozygote" targets. Therefore, unambiguous target allele assignment of 1- to 4-bp indel homozygotes and heterozygotes could be achieved by employing either, and only one, hybridization probe. In this respect, DASH analysis of short indels is essentially equivalent to genotyping SNPs by DASH.

When DASH was modeled on the 5bp indel, the matched probe-target duplexes (i.e., insertion probe plus insertion target, or deletion probe plus deletion target) generated typical hightemperature $\mathrm{T}_{\mathrm{m}}$ peaks (Figure 1E). The 


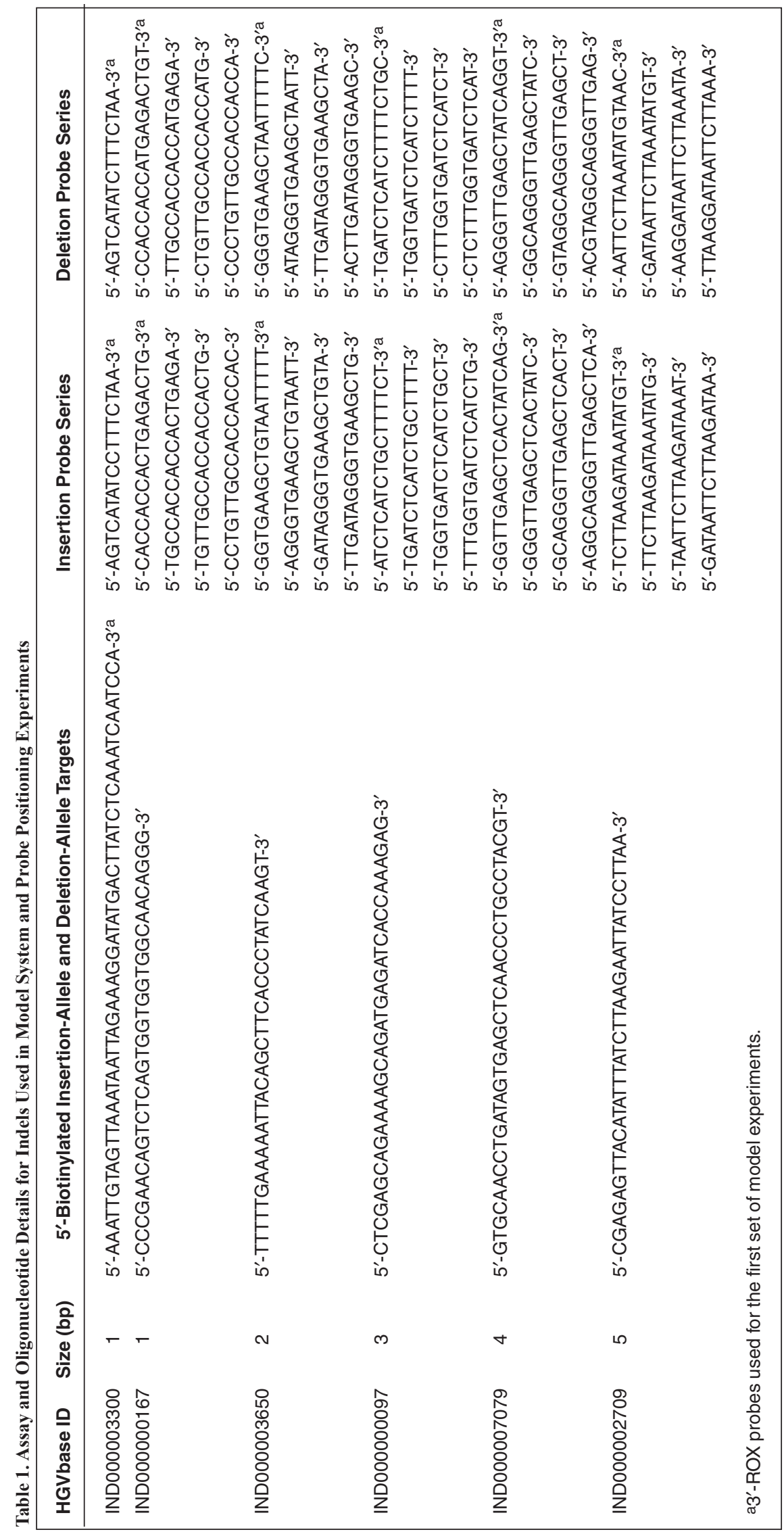

deletion probe tested against the insertion target generated a mismatched signal $\mathrm{T}_{\mathrm{m}}$ that was about $10^{\circ} \mathrm{C}$ lower than when this probe was used with its matched deletion target; when testing the artificial heterozygous mixture with the deletion probe, the expected dual peaks were produced. However, the 5bp insertion probe tested against its mismatched deletion target generated a $T_{m}$ that was so low that it was barely discernable within the standard DASH assay window $\left(35^{\circ}-80^{\circ} \mathrm{C}\right)$. Consequently, the mock heterozygote signal from the 5-bp insertion probe appeared very similar to the result obtained when testing just the insertion target.

Across all of our studies, we noted that mismatched target-probe duplexes were much less stable when the inserted DNA bases reside within the probe rather than in the target, as highlighted by our findings with the 5-bp insertion probe described above. Specifically, $(i)$ relative to $T_{m} s$ produced by matched probe-target duplexes, all insertion probe mismatch $\mathrm{T}_{\mathrm{m}} \mathrm{s}$ were decreased by a greater degree than were the deletion probe mismatch $\mathrm{T}_{\mathrm{m}} \mathrm{s}$; and (ii) mismatch $\mathrm{T}_{\mathrm{m}} \mathrm{s}$ tended to be lower (relative to their matched $\mathrm{T}_{\mathrm{m}} \mathrm{s}$ ) for larger indels. There are fewer base-paired residues in the case of the insertion probe-deletion target duplex, and these differences must influence the relative ease with which the two duplexes can be denatured by increasing temperature. Longer allelic differences tend to destabilize the mismatched probe-target duplexes (particularly those involving the insertion probe), such that only matched probetarget $T_{m}$ s can be reliably detected. In this case, the assay is generating a simple "plus-minus" signal, which is indicative of the presence or absence of target DNA matched to the probe, while giving no information about the presence or absence of the alternative target allele. Hence, for longer indels, using either probe alone would not necessarily allow us to readily distinguish heterozygotes from matched homozygotes (insertion probe, insertion target and deletion probe, and deletion target duplexes), nor would it discriminate between failed PCRs and mismatched homozygotes (insertion target, deletion probe and deletion target, insertion probe duplexes). It is possible that by 


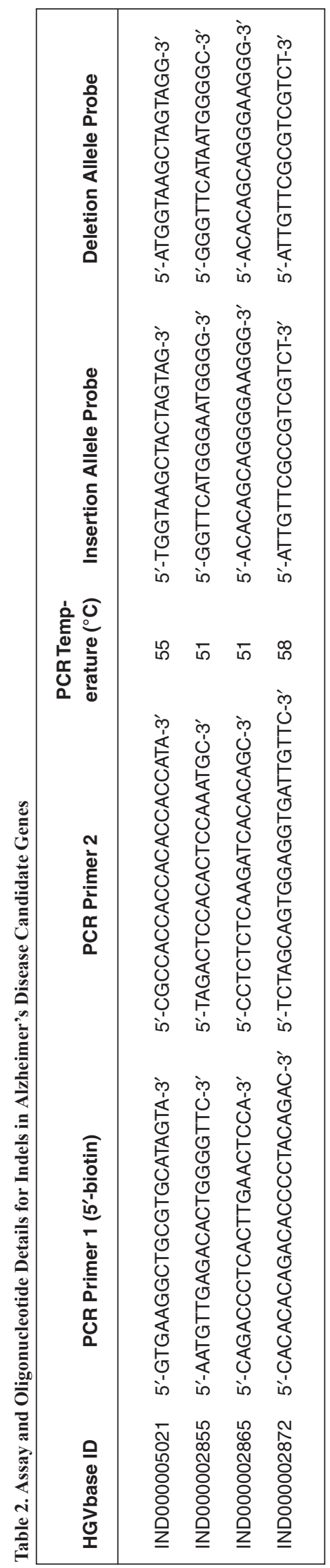

296 BioTechniques increasing the length of the probes we would also increase the annealing temperature sufficiently to visualize the insertion target, deletion probe peak, but this has not been explored. Thus, for longer indels, we would preferably utilize both probes in a serial manner.

\section{Model Experiments-Probe Positioning}

We extended the model system to explore alternative placements of the probes relative to the indel sequences. For one example of each 1- to 5-bp indel mock assay described above, three additional insertion probes and three additional deletion probes were synthesized. These 17- to 18-bp probe sequences were designed to span regions staggered progressively $5^{\prime}$ (relative to the probe strand) in 1-3 nucleotide steps, up to and including the extreme situation where the indel sequence resided at the very end of the probe (Figure 3 ).

Each probe was used in duplicate DASH experiments to interrogate its insertion target and its deletion target. The general finding was that the relative destabilization of the mismatched probe-target duplexes was progressively lessened the further off-center the probe was placed. That is, for each indel sequence, the presence of an increasingly long uninterrupted stretch of perfectly matched probe-target bases to one side of the mismatched region led to increasingly higher temperature-mis-

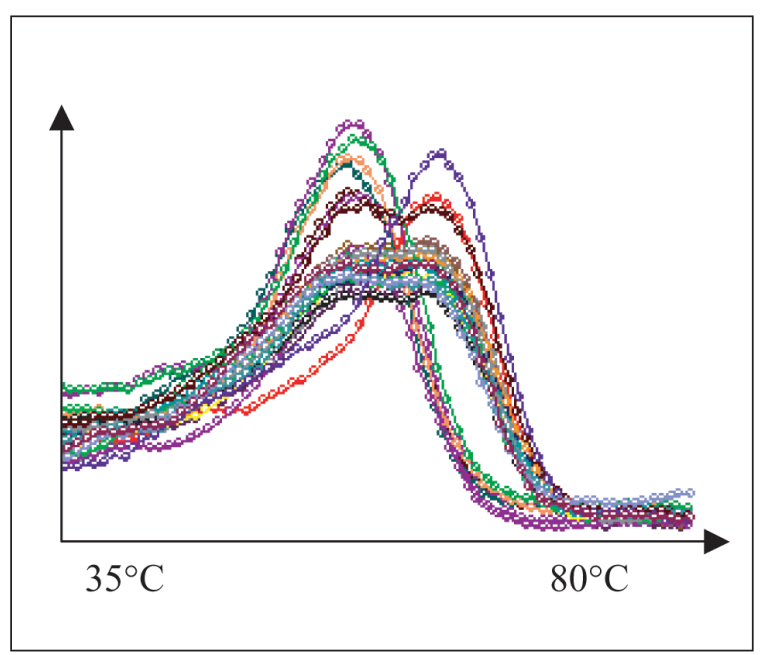

Figure 2. Multiple genotyping peaks from IND000002872. Peaks show a uniform pattern, with $6^{\circ} \mathrm{C}$ separating the match and mismatch peaks. The y-axis is -dFluorescence/dTemperature. matched $T_{m}$ peaks. Consequently, all the windows of separation between $\mathrm{T}_{\mathrm{m}} \mathrm{s}$ from matched versus mismatched probe-target duplexes (as described above for centrally placed probes) became too small to be used for reliable target allele discrimination when the most off-center probes were employed. This was true even in the case of longer indel insertion probes, which had been found to be particularly unstable when centrally located. A slight offset for these probes did bring the mismatched $\mathrm{T}_{\mathrm{m}} \mathrm{s}$ into a measurable range to create a usable $T_{m}$ discrimination window, but this would be difficult to predict or utilize in experiments without empirical testing for different indel sequences. Consequently, the most robust general approach to designing indel assays entails placing the probe centrally over the polymorphic position and using the deletion probe either alone or serially with the insertion probe.

\section{Scoring Indels in Genomic DNA Samples}

To test the model system conclusions in a genotyping experiment from DNA rather than synthetic oligonucleotides, we undertook a series of Alzheimer's disease association studies using four indels from two candidate disease genes (APBB1 and LRP1). These studies entailed genotyping 277 Alzheimer's disease cases and control individuals. The HGVbase IDs and sizes for the indels used in this study are as follows: IND000002855 (3 bp), IND000002865 (1 bp), IND000002872 (1 bp), and IND000005021 (3 bp). Table 2 outlines the DASH assay details.

Precisely as predicted by the model system, the 1-bp indels generated clearly distinct $T_{m}$ peaks for the two target alleles when interrogated by the insertion probe or by the deletion probe (Figure 2a). Likewise, the 3-bp indels behaved as predicted in that the mismatched $\mathrm{T}_{\mathrm{m}} \mathrm{s}$ were far lower for the insertion probes than for the deletion probes. In 
Table 3. Results from Association Study Analysis of Indels in Alzheimer's Disease Candidate Genes

\begin{tabular}{|lllll|}
\hline Gene Name & APBB1 & LRP1 & LRP1 & LRP1 \\
\hline HGVbase ID & IND000005021 & IND000002855 & IND000002865 & IND000002872 \\
Indel & TAG/--- & GGG/--- & C/- & G/- \\
Allele Frequency (Cases) & $(---)=21 \%$ & GGG=43\% & C=29\% & G=31\% \\
Allele Frequency (Controls) & $(---)=16 \%$ & GGG=36\% & C=31\% & G=33\% \\
Hardy Weinberg Equilibrium & Yes & Yes & Yes & Yes \\
Pvalue of Assocation to & 0.06 & 0.70 & 0.63 & 0.80 \\
Alzheimer's disease & & & & \\
\hline
\end{tabular}

\section{5' XXXXXXXXXXXXXXXXXXXXXXXXVXXXXXXXXXXXXXXXXXXXXXXXX3' 3' YYYYYYYYVYYYYYYYY5' \\ 3' YYYYYVYYYYYYYYYYY5' \\ 3' YYVYYYYYYYYYYYYYY 5' \\ 3'vYYYYYYYYYYYYYYYY5'}

Figure 3. Alternative probe positioning. X represents the target DNA, Y represents the probe DNA, and $v$ represents the DNA variation (an indel sequence in this case). Four example probes are shown in register with where they would hybridize. Their sequences are progressively staggered away from the central position until the polymorphic DNA is at the $3^{\prime}$ end of the probe.

one case (IND000005021), the insertion probe yielded a plus-minus scoring pattern, while the insertion probe for the other 3-bp indel (IND000002855) gave rise to a mismatched peak that could just be read at the lower end of the standard DASH assay window $\left(35^{\circ}-80^{\circ} \mathrm{C}\right)$. Although in the model systems we did not see a plus-minus scoring pattern for the 3-bp indel, the range between $T_{m} \mathrm{~s}$ for the match and the mismatch are clearly dependent on the sequence of the target.

The genotype frequencies for these Alzheimer's disease candidate gene indels were found to be in HardyWeinberg equilibrium in both cases and controls. $P$ values for the HardyWeinberg equilibrium are as follows: IND000005021, 0.71; IND000002865, 1.0; IND000002855, 1.0; and IND$000002872,1.0$. None of the indels in candidate genes showed significant disease association (Table 3), suggesting that these polymorphisms are not related to Alzheimer's disease in the tested population.

In conclusion, after testing 10 different 1- to 5-bp indel polymorphisms, we find that DASH is an effective and robust system for genotyping this form of sequence variation. Therefore, it can now be recommended as a proven method not only for SNP genotyping but also for broad use in human indel polymorphism analysis.

\section{ACKNOWLEDGMENTS}

We gratefully acknowledge the Carl Tryggers Foundation for Scientific Research and The Swedish Vetenskapsrådet for funding that helped with aspects of our research undertakings. We also thank the Center for Genomics and Bioinformatics at the Karolinska Institute for provision of enabling infrastructure.

\section{REFERENCES}

1.Weber, J.L., D. David, J. Heil, Y. Fan, C. Zhao, and G. Marth. 2002. Human diallelic Hum. Genet. 71:854-862.

2.Dawson, E., Y. Chen, S. Hunt, L.J. Smink, A. Hunt, K. Rice, S. Livingston, S. Bumpstead, et al. 2001. A SNP resource for human chromosome 22: extracting dense clusters of SNPs from the genomic sequence. Genome Res. 11:170-178.

3.Fredman, D., M. Siegfried, Y.P. Yuan, P. insertion/deletion polymorphisms. Am. J.
Bork, H. Lehvaslaiho, and A.J. Brookes. 2002. HGVbase: a human sequence variation database emphasizing data quality and a broad spectrum of data sources. Nucleic Acids Res. 30:387-391.

4.Prince, J.A., L. Feuk, W.M. Howell, M. Jobs, T. Emahazion, K. Blennow, and A.J. Brookes. 2001. Robust and accurate single nucleotide polymorphism genotyping by dynamic allele-specific hybridization (DASH): design criteria and assay validation. Genome Res. 11:152-162.

5.Woodburn, K. and E. Johnstone. 1999. Ascertainment of a population of people with early-onset dementia in Lothian, Scotland. Int. J. Geriatr. Psychiatr. 14:362-367.

6.Folstein, M.F., S.E. Folstein, and P.R. McHugh. 1975. "Mini-mental state". A practical method for grading the cognitive state of patients for the clinician. J. Psychiatr. Res. 12:189-198.

7.Howell, W.M., M. Jobs, and A.J. Brookes. 2002. iFRET: an improved fluorescence system for DNA-melting analysis. Genome Res. 12:1401-1407.

Received 21 March 2003; accepted 14 April 2003.

Address correspondence to Anthony $J$. Brookes, Center for Genomics and Bioinformatics, Karolinska Institute, Berzeliusväg 35, S-171 77 Stockholm, Sweden. e-mail: anthony.brookes@cgb.ki.se 